\title{
Preliminary Evaluation of Possibilities for Determination of Optical Density of Venous Blood Plasma for Diagnosis in Emergency Abdominal Surgery
}

\author{
Andrii I Shurma $^{1 *}$, Fedir V Grynchuk ${ }^{1}$ and Artem V Motric ${ }^{2}$ \\ ${ }^{1}$ Department of Surgery № 1, Bukovinian State Medical University, Chernivtsi, Ukraine \\ ${ }^{2}$ Department of Optics and Printing, Yuriy Fedkovych Chernivtsi National University, \\ Chernivtsi, Ukraine \\ *Corresponding Author: Andrii I Shurma, Department of Surgery № 1, Bukovinian \\ State Medical University, Chernivtsi, Ukraine.
}

Received: September 08, 2021

Published: September 23, 2021

(C) All rights are reserved by Andrii I Shurma., et al.

\section{Abstract}

Background/Aims: The basis of laboratory diagnosis in emergency abdominal surgery is to determine the number of leukocytes and their forms, the content of C-reactive protein. But the results of such studies are nonspecific. With unclear clinical symptoms, this creates the preconditions for diagnostic errors. Therefore, the search for new diagnostic tools that would allow for a quick, accurate assessment remains relevant. Therefore, the aim of the study was to pre-evaluate the diagnostic possibilities of determining the optical density of venous blood plasma in the infrared range for emergency abdominal surgery.

Methods: 42 patients were examined. Among them were: liver abscess - 4 cases, acute phlegmonous appendicitis with periappendicular abscess - 4, acute phlegmonous appendicitis with diffuse peritonitis - 5, acute phlegmonous appendicitis without complications - 8, acute phlegmonous cholecystitis without complications - 5, perforation of gastroduodenal ulcer with diffuse peritonitis - 5, acute adhesive intestinal obstruction without complications - 4, acute catarrhal appendicitis without complications - 3, acute catarrhal cholecystitis without complications - 4 cases.

At patients on admission to hospital venous blood from an ulnar vein was collected. Measured the optical density of venous blood plasma on a spectrophotometer SF-4A in the wavelength range $\lambda=2.0-20.0 \mu \mathrm{m}$. The measurement results were compared with the results of the removed organs histological examination.

The control group consisted of 11 patients who had no destructive changes in organs and complications: 4 patients with adhesive intestinal obstruction, 3 patients with acute catarrhal appendicitis, 4 patients with acute catarrhal cholecystitis without complications.

Results: We found in the infrared spectrum a number of maxima of the values of the blood plasma optical density, which are localized at wavelengths $\lambda=3.1,3.5,4.3,6.1,6.7,9$ and $17.5 \mu \mathrm{m}$. Indicators of optical density at these wavelengths differed in different diseases and complications.

For analysis and comparison, patients were also divided into groups: with abscesses, with diffuse peritonitis, with phlegmonous changes of organs without complications (appendicitis, cholecystitis) and control (no organ destruction). In this comparison, it was found (Figure) that at wavelengths $\lambda=3.1,6.1,6.7,9$ and $17.5 \mu \mathrm{m}$, the optical density indices differ with specific regularities. In particular, each time in patients from the control group the parameters were statistically significantly $(p<0.05)$ lower than in patients with inflammatory-destructive pathology. In addition, the indicators differ in patients with diffuse peritonitis, abscesses and uncom- 
plicated phlegmonous appendicitis and cholecystitis.

Conclusion: 1. Patients with acute surgery have specific maxima of optical density of venous blood plasma in the infrared region at wavelengths $\lambda=3.1,3.5,4.3,6.1,6.7,9$ and $17.5 \mu \mathrm{m}$. 2. Indicators of optical density of blood plasma at wavelengths $\lambda=3.1,6.1$. 6.7, 9, 17.5 microns differ, depending on features of the main disease and complications and have the general laws at perforation of hollow bodies, abscesses, diffuse peritonitis, phlegmonous changes of bodies without complications. 3 . The study of optical density is a promising area for improving the diagnosis in emergency abdominal surgery.

\section{Introduction}

The basis of laboratory diagnosis in emergency abdominal surgery is to determine the number of leukocytes and their forms, the amount of C-reactive protein [1-6]. But the results of such examination are nonspecific. With unclear clinical symptoms, this creates the preconditions for diagnostic errors [7]. Therefore, in case of diagnostic difficulties, instrumental methods are used [1-7]. Such methods may require special training, invasive manipulations, etc. This reduces the possibilities of their use, so the attitude to such methods is ambiguous [1,5,7-10]. Therefore, the search for new diagnostic tools that would allow a quick, accurate assessment remains relevant. The study of the optical characteristics of blood attracts attention, which are used to diagnose both individual diseases and their complications [11,12].

\section{Aim of the Study}

The aim of the study was to pre-evaluate the diagnostic possibilities of determining the optical density of venous blood plasma in the infrared range for emergency abdominal surgery.

\section{Materials and Methods}

42 patients were examined. Among them were: liver abscess - 4 cases, acute phlegmonous appendicitis with periappendicular abscess - 4, acute phlegmonous appendicitis with diffuse peritonitis 5 , acute phlegmonous appendicitis without complications -8 , acute phlegmonous cholecystitis without complications - 5, perforation of gastroduodenal ulcer with diffuse peritonitis -5 , acute adhesive intestinal obstruction without complications - 4, acute catarrhal appendicitis without complications - 3 , acute catarrhal cholecystitis without complications - 4 cases.

At patients on admission to hospital venous blood from an ulnar vein was collected. Measured the optical density of venous blood plasma on a spectrophotometer SF-4A in the wavelength range $\lambda$
$=2.0-20.0 \mu \mathrm{m}$. The measurement results were compared with the data of histological examination of the removed organs.

The control group consisted of 11 patients who did not have destructive changes in organs and complications: 4 patients with adhesive intestinal obstruction, 3 with acute catarrhal appendicitis, 4 with acute catarrhal cholecystitis without complications.

The hypothesis of normal data distribution (Gaussian distribution) was tested in selections by Shapiro-Wilk criterion. Verification of the hypothesis of average data equality was carried out by Wilcoxon criterion. The results of the study were statistically processed by the Microsoft ${ }^{\circledR}$ Office Excel (build 11.5612.5703) tables. Statistical significance was set at $\mathrm{p}<0.05$ for all analyses.

\section{Results and Discussion}

We found in the infrared spectrum a number of maxima of the values of the optical density of blood plasma, which are localized at wavelengths $\lambda=3.1,3.5,4.3,6.1,6.7,9$ and $17.5 \mu \mathrm{m}$ (Table). Indicators of optical density at these wavelengths differed in different diseases and complications.

These results indicate that the optical density of venous blood plasma changes, depending on the characteristics of intra-abdominal pathology. In particular, in the presence of liver abscesses and periappendicular abscess, the parameters of the indicators are clearly different from the rest. Indicators in patients with common forms of peritonitis due to perforation of hollow organs also have common patterns. Certain common differences are also noted in patients with phlegmonous appendicitis and cholecystitis. In general, the indicators in patients without destructive intra-abdominal pathology (control) differ at most wavelengths.

For analysis and comparison, patients were also divided into groups: with abscesses, with diffuse peritonitis, with phlegmon- 
ous changes of organs without complications (appendicitis, cholecystitis) and control (no organ destruction). In this comparison, it was found (Figure) that at wavelengths $\lambda=3.1,6.1,6.7,9$ and $17.5 \mu \mathrm{m}$, the optical density indices differ with specific regularities. In particular, each time in patients from the control group the parameters were statistically significantly $(\mathrm{p}<0.05)$ lower than in patients with inflammatory-destructive pathology. In addition, the indicators differ in patients with diffuse peritonitis, abscesses and uncomplicated phlegmonous appendicitis and cholecystitis.

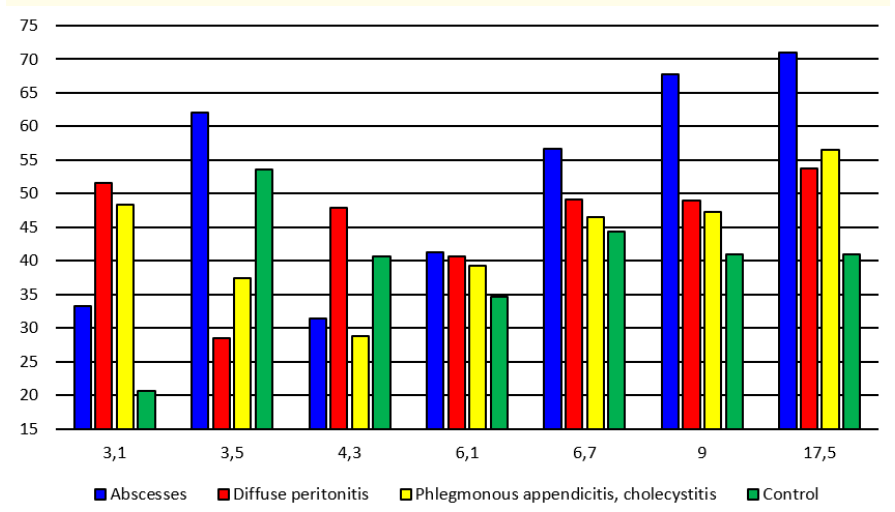

Figure 1: Indicators of optical density of venous blood plasma, depending on the nature of the underlying disease and the presence of complications.

Note that the small number of observations currently does not allow to draw definitive conclusions. However, the preliminary results of the study suggest that the determination of the optical density of venous blood plasma in the infrared spectrum may be a promising area for improving the diagnosis in emergency abdominal surgery. Therefore, in our opinion, such studies need to be continued.

Such studies can be used to differentiate individual diseases and their complications, as well as to diagnose the presence of destructive changes in the body (appendix, gallbladder). Note also the simplicity and accessibility of the method. The measurement takes a few minutes.

The question of the reasons and mechanisms of development of differences of indicators for their correct interpretation is also natural. According to the literature [1], changes in the parameters of the optical density of blood plasma may be a reflection of changes in the activity of immune mechanisms. However, this issue requires further research, including experimental.

\section{Conclusion}

1. In patients with abdominal acute surgery there are specific maxima of optical density of venous blood plasma in the infrared region at wavelengths $\lambda=3.1,3.5,4.3,6.1,6.7,9$ and $17.5 \mu \mathrm{m}$.

2. Indicators of optical density of blood plasma at wavelengths $\lambda$ $=3.1,6.1$. 6.7, 9, 17.5 microns differ, depending on features of the basic disease and complications and have the general laws at perforation of hollow body organs, abscesses, diffuse peritonitis, phlegmonous changes without complications.

3. The study of optical density is a promising area for improving the diagnosis in emergency abdominal surgery.

\section{Authors' Contribution}

A.I. Shurma: Obtaining data for analysis, writing the text of an article.

F.V. Grynchuk: Research design development, data analysis.

A.V. Motric: Optical density measurement.

\section{Funding Support}

This study required no funding.

\section{Conflict of Interest}

The authors declare that they have no conflict of interest.

\section{Bibliography}

1. Di Saverio S., et al. "Diagnosis and treatment of acute appendicitis: 2020 update of the WSES Jerusalem guidelines". World Journal of Emergency Surgery 15.27 (2020).

2. Rushing A., et al. "Management of acute appendicitis in adults: A practice management guideline from the Eastern Association for the Surgery of Trauma". Journal of Trauma and Acute Care Surgery 87.1 (2019): 214-224.

3. Leppäniemi A., et al. "WSES guidelines for the management of severe acute pancreatitis". World Journal of Emergency Surgery $15.3(2020)$. 
4. Tarasconi A., et al. "Perforated and bleeding peptic ulcer: WSES guidelines”. World Journal of Emergency Surgery 15 (2020): 3.

5. Pisano M., et al. "World Society of Emergency Surgery updated guidelines for the diagnosis and treatment of acute calculus cholecystitis". World Journal of Emergency Surgery 15.61 (2020).

6. Hecker A., et al. "Intra-abdominal sepsis: new definitions and current clinical standards". Langenbeck's Archives of Surgery 404 (2019): 257-271.

7. Gans SL., et al. "Guideline for the diagnostic pathway in patients with acute abdominal pain". Digestive Surgery 32.1 (2015): 23-31.

8. Patriti A., et al. "Emergency general surgery in Italy during the COVID-19 outbreak: first survey from the real life". World Journal of Emergency Surgery 15.1. (2020): 36.

9. Liv Bjerre Juul Nielsen., et al. "Laparoscopy in major abdominal emergency surgery seems to be a safe procedure". Danish Medical Journal 64.5. (2017): A5370.

10. Catena F., et al. "Bowel obstruction: a narrative review for all physicians". World Journal of Emergency Surgery (2019).

11. Kuyun LA. "Differential diagnostics of different forms of peritonitis by means of Chemiluminescense". Bulletin of Problems in Biology and Medicine 2.138 (2017): 21-24.

12. Grynchuk FV., et al. "Experimental justification of definition of optical density plasma venous blood for diagnostik of intraabdominal inflammatory processes". Clinical and Experimental Pathology 10.1. (2011): 38-40.

Volume 4 Issue 10 October 2021

(C) All rights are reserved by Andrii I Shurma., et al. 